Available online on 15.05.2020 at http://jddtonline.info
Open Access to Pharmaceutical and Medical Research
unrestricted non-commercial use, provided the original work is properly cited

Open@ Access

Research Article

\title{
Efficient Enhancement in Itraconazole Solubility through its Cyclodextrin- Water Soluble Polymer Ternary Inclusion Complexes
}

\author{
Sid Dounia ${ }^{1, *}$, Baitiche Milad ${ }^{1}$, Elbahri Zineb ${ }^{2}$, Djerboua Ferhat ${ }^{1}$, Messalti Sabrine ${ }^{1}$, Boutahala Mokhtar ${ }^{3}$ \\ 1 Laboratoire de préparation, modification et applications des matériaux polymériques multiphasiques, Département de Génie des procédés, \\ Faculté de technologie, Université Ferhat Abbas Sétif-1, Boulevard du Sipion, Sétif 19000, Algérie \\ 2 Laboratory of materials and catalysis, Faculty of Exact Sciences, Djillali Liabès University of Sidi Bel Abbès, 22000 Algeria \\ 3 Laboratoire de génie des procédés chimiques, Département de Génie des procédés, Faculté de technologie, Université Ferhat Abbas Sétif-1, \\ Boulevard du Sipion, Sétif 19000, Algérie
}

\begin{abstract}
The aim of this work is the enhancement of the hydrosolubility behaviour of a poorly soluble, weakly basic drug, using itraconazole (ITZ) as a case example. Binary inclusion complexes of ITZ with $\beta$-cyclodextrin ( $\beta$-CD) are prepared in $1: 2$ molar ratios of ITZ to $\beta$-CD by co-evaporation method. Both solubility and dissolution behaviour are compared with that of the pure drug. Ternary complexes can be obtained by adding the polyvinylpirrolidone (PVP) which is a highly water soluble polymer, in the ITZ/ $\beta$-CD complex formation. Actually, Solid state analysis is performed for all formulations and for pure ITZ applying the Fourier transforms infrared (FT-IR) spectroscopy, powder X-ray diffraction (pXRD) and differential scanning calorimetry (DSC). Solubility tests indicate that with all formulation, the solubility of ITZ formed with $\beta$-CD or $\beta$ CD and PVP proved to be increased. The obtained results show that the pure drug has a poor dissolution property, and the ternary inclusion complexes resulted in fast and extensive release of ITZ.
\end{abstract}

Keywords: Itraconazole, $\beta$-cyclodextrin, polyvinylpyrrolidone.

Article Info: Received 24 Feb 2020; Review Completed 02 April 2020; Accepted 12 April 2020; Available online 15 May 2020

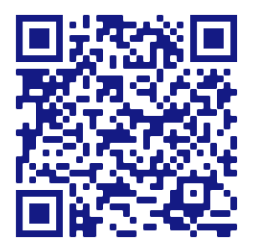

Cite this article as:

Sid D, Baitiche M, Elbahri Z, Djerboua F, Messalti S, Boutahala M, Efficient Enhancement in Itraconazole Solubility through its Cyclodextrin-Water Soluble Polymer Ternary Inclusion Complexes, Journal of Drug Delivery and Therapeutics. 2020; 10(3):11-14 http://dx.doi.org/10.22270/jddt.v10i3.4046

Sid Dounia, Laboratoire de préparation, modification et applications des matériaux polymériques multiphasiques, Département de Génie des procédés, Faculté de technologie, Université Ferhat Abbas Sétif-1, Boulevard du Sipion, Sétif 19000, Algérie.

\section{INTRODUCTION}

Itraconazole is a triazole antifungal agent that exhibits a broad spectrum of activity with remarkable tolerance. Itraconazole is highly effective, due to its main metabolite namely: hydroxy-itraconazole that shows an important antifungal activity. It belongs to the Biopharmaceutical Classification System (BCS) class II and shows poor aqueous solubility (1 ng/ml) (1). The poor inherent drug solubility and slow dissolution rate in the gastrointestinal tract engender some difficulties in manufacturing suitable pharmaceutical formulations. In addition, it may also lead to low oral bioavailability and irreproducible clinical response of the drug (2).

In the last few decades, the obtained data confirm that the bioavailability of BCS class II drugs can be improved by enhancing their solubility profiles and dissolution characteristics (3). Various approaches like particle size ISSN: 2250-1177 reduction (4), drug dispersion in carrier (5), modification of crystal habit (6), use of surfactants (7), self-emulsifying formulations (2), formation of water-soluble inclusion complexes (8) etc. are investigated to improve the dissolution profile of the poorly soluble drugs. Among them, the complexation with cyclodextrins (CDs) represents one of the advanced techniques (9). CDs are structurally interrelated oligosaccharides with six $(\alpha-C D)$, seven $(\beta-C D)$ and eight $(\gamma$-CD) $\alpha-1,4$ - glucopyranose units. They have a lipophilic core with hydrophilic outer surface allows noncovalent inclusion complexes formation with hydrophobic drugs. This fact, is obtained by taking up all or some part of the moiety into the cavity [8]. These inclusion complexes are revealed to enhance the guest bioactive molecules apparent stability, solubility, dissolution rate and bioavailability. The $\beta-C D$ is the most frequently used pharmaceutical cyclodextrins excipient due to its high availability, low cost, excellent biocompatibility, considerable 
regulatory acceptance and preferred cavity dimension (1012). Note that naturally occurring cyclodextrins ( $\alpha$ - and $\gamma$ CD) are expensive relative to $\beta$-CD. Furthermore, recent report mention that $\beta$-CD derivative such HP- $\beta$-CD is more toxicologically benign than the natural $\beta$-CD (13),(14). Pioneering studies in this field show that one can increase complexation and solubilizing efficiencies of the $\beta$-CD (with less formulation bulk), by adding small quantities of a suitable hydrophilic polymer to a drug- $\beta-C D$ system (13). These obtained results are due to the synergistic effect of polymer and $\beta$-CD on the formation of ternary complexes or co-complexes. The water-soluble polymers can also increase the apparent stability constant of the inclusion complexes (15). In fact, this work is an experimental demonstration of the credibility of novel inclusion complexes of the ITZ with $\beta$ $\mathrm{CD}$ in absence and presence of polyvinylpyrrolidone (PVPK30) in order to improve the solubility and ITZ dissolution. I Actually, we investigate the formation of complexes between ITZ and $\beta$-CD and the effect of hydrophilic polymer (PVP-30) on complexation and solubilizing efficiency. In fact, Infrared spectroscopy (IR), differential scanning calorimetry (DSC) and X-ray diffraction (XRD) are carried out in order to characterize the ITZ and its inclusion complexes. Finally, the release of the drug from binary and ternary complexes at $\mathrm{pH}$ 1.2 are also studied using the UV-VIS spectroscopy.

\section{MATERIALS AND METHODS}

\section{MATERIALS}

ITZ and PVP-K30 are a gift sample from of SALEM Laboratory El- EULMA (Algeria). $\beta$-CD is purchased from SIGMA-ALDRICH, USA. All the other chemicals and solvents used are of analytical grade and procured commercially.

\section{Binary and Ternary Inclusion Complexes Preparation}

In a preliminary set of experiments, the optimal ITZ- $\beta-C D$ ratio is determined in phase solubility studies. In fact, the obtained results from these studies indicate that the optimal ITZ- $\beta$-CD ratio is $1: 2$. Note that the molar ratio is adopted in this study. Ternary complexes are made by combining PVP$\mathrm{K} 30$ at the concentration $10 \%$ of the solid complex.

In the sequel, we explain the inclusion complexes steps. At the beginning, we dissolve $705 \mathrm{mg}$ ITZ in a mixture of 100 $\mathrm{mL}$ ethanol $96 \%$ and $\mathrm{HCl}$ conc (ratio $10: 1$ ) at $50^{\circ} \mathrm{C}(5)$. Then, in a different container, an appropriate amount of $\beta$-CD (or, appropriate amounts of the $\beta$-CD in the case of ternary complex preparation) and PVP-K30 are dissolved in distilled water. Furthermore, the ITZ solution is mixed with the excipient solution and the resulting mixture is stirred for $2 \mathrm{~h}$ until getting a clear solution. Then, the solution is evaporated under vacuum at temperature of $45^{\circ} \mathrm{C}$ and $100 \mathrm{rpm}$ in a rotary evaporator (BUCHI, ROTAVAPOR R-215). The solid residue is further dried completely at $50^{\circ} \mathrm{C}$ for 24 hours. Finally, dried complex is pulverized into a fine.

\section{Fourier Transform Infrared (FT-IR) Spectroscopy}

The infrared spectra of the samples is obtained with an IR Affinity-1S, SHIMADZU spectrometer using the potassium bromide (KBr) disk technique (1 $2 \% \mathrm{w} / \mathrm{w}$ samples in $\mathrm{KBr})$, They are prepared with a hydrostatic press at a force of $10 \mathrm{~T}$ $\mathrm{cm}^{-2}$ for $3 \mathrm{~min}$. Note that the used interval of scanning is $4000-400 \mathrm{~cm}^{-1}$

\section{Differential Scanning Calorimetry}

Differential Scanning Calorimetry (DSC) measurements for pure drug, $\beta-C D$ and inclusion complexes are performed using a DSC SETARAM instrument. After being heating under nitrogen flow $(20 \mathrm{ml} / \mathrm{min})$ at a scanning rate of $10^{\circ} \mathrm{C} \mathrm{min}-1$ and a temperature range of $25^{\circ} \mathrm{C}$ to $350^{\circ} \mathrm{C}$, the samples between (10-15 mg) are put in sealed aluminum pans. However, an empty aluminium pan is consider as reference.

\section{X-Ray Powder Diffraction (XRPD)}

X-ray powder diffractograms of individual components and the complex systems are obtained on a PAN analytical kind X' PERTPRO Diffractometer. The used radiations are generated by a copper filter, with wavelength $1.54 \mathrm{~A}^{\circ}$ at $40 \mathrm{kV}$ and 30 $\mathrm{mA}$. From the other hand, the Glass slide are covered with the sample in order to be analyzed and scanned over a range from $1^{\circ}$ to $60^{\circ} 2 \theta$ degrees, using a scan rate of 1 degree per min and a steps scan of 0.02 .

\section{Drug Content}

The drug content in the complexes obtained are determined by extraction using a SHIMADZU-1800 UV-visible spectrophotometer. A known weight of each complex (50 $\mathrm{mg}$ ) are placed in a $50 \mathrm{~mL}$ volumetric flask, and pure ethanol are added. The concentration of ITZ in the solution are determined by using the standard curve of a series of standard solutions of known ITZ concentrations. Extractions are carried out in triplicate and the drug content in the complex are calculated using the following formula:

ITZ content in complex $(\%)=\left(\frac{\text { mass of ITZ extracted }}{\text { mass of complex }}\right) \times 100$

\section{In Vitro Release Tests}

The in vitro release test of ITZ from all drug-carrier systems, and for ITZ alone was conducted using the United States Pharmacopeia Paddle Method (Apparatus II) on an Heidolph RZR 2041, an amount of each samples equivalent to $25 \mathrm{mg}$ of ITZ is placed into a hard gelatin capsule, and then, soaked into $900 \mathrm{~mL}$ of simulated gastric medium $(0.1 \mathrm{~N} \mathrm{HCl} \mathrm{pH} \mathrm{1.2)}$ for $2 \mathrm{~h}$. The dissolution media maintained at $37 \pm 0.5^{\circ} \mathrm{C}$ and stirred at $100 \mathrm{rpm}$.

At suitable ranges, $5 \mathrm{~mL}$ of the dissolution medium are withdrawn, using a syringe, then, filtered through $0.45 \mu \mathrm{m}$ nylon disc filter, and equivalent volumes of fresh medium are added to maintain the sink condition. The ITZ content is determined at $282 \mathrm{~nm}$ using an UV-VIS spectrophotometer. Note that, each dissolution test is performed in duplicate.

\section{RESULTS AND DISCUSSION}

\section{FTIR Analysis}

For analysing the plausible interactions among ITZ, $\beta$-CD and PVPK30 in the solid state, the IR spectra of binary and ternary complexes are compared to that of pure drug (Fig. 1). The IR spectrum of ITZ is characterized by principal absorption peaks at a wavelength of $3325 \mathrm{~cm}-1$ and 3111 cm-1 match to the absorption of NH2 group. Actually, Sharp peak at a waverlength of $1699 \mathrm{~cm}-1$ is due to vibrations of $\mathrm{C}$ $=0$ groups. The characteristic peak in the visible waver length of $1442 \mathrm{~cm}-1$ and $1508 \mathrm{~cm}-1$ related to the links C-H (16).

The $\beta$-CD frequencies such $3331 \mathrm{~cm}-1,2931 \mathrm{~cm}-1,1153$ $\mathrm{cm}-1$, and $1028 \mathrm{~cm}-1$, correspond to the symmetric and asymmetric stretching of $\mathrm{O}-\mathrm{H}, \mathrm{CH} 2, \mathrm{C}-\mathrm{C}$ and bending $\mathrm{O}-\mathrm{H}$ vibration, respectively (17). The most important peaks of PVP-K30 are the stretching vibration of the carbonyl (CO) and amide $(\mathrm{N}-\mathrm{H})$ groups that characteristically appeared around $1678 \mathrm{~cm}-1$ and $1510 \mathrm{~cm}-1$, respectively (5). The FTIR spectra of both binary and ternary complexes exhibited some considerable shifting in the characteristic bands. The inclusion of the aromatic ring into the electron rich cavity of $\beta$-CD amplified the density of electron cloud, which leaded to 
the increase in frequency. The hydrogen bonding contacts and Van der Waals forces in complexes may alter in the microenvironment, which probably decreases the frequency between the inclusion complex and its constituent molecules (18). We note that, the intensity of the skeletal bands is attenuated in the IR spectra of both the binary and ternary systems. In fact, it suggested the entire or partial entrapment of ITZ into the host cavity at the phase of inclusion complexation (8). However, the absorption band of amide band is much narrower for the ternary mixture case. Actually, we note an important impact of the potential implication of the amide group of PVP-K30 in the complexation process (9). Overall, the binary and ternary inclusion complexes do not display any new peaks meaning that no chemical bonds are created in the formed complexes.

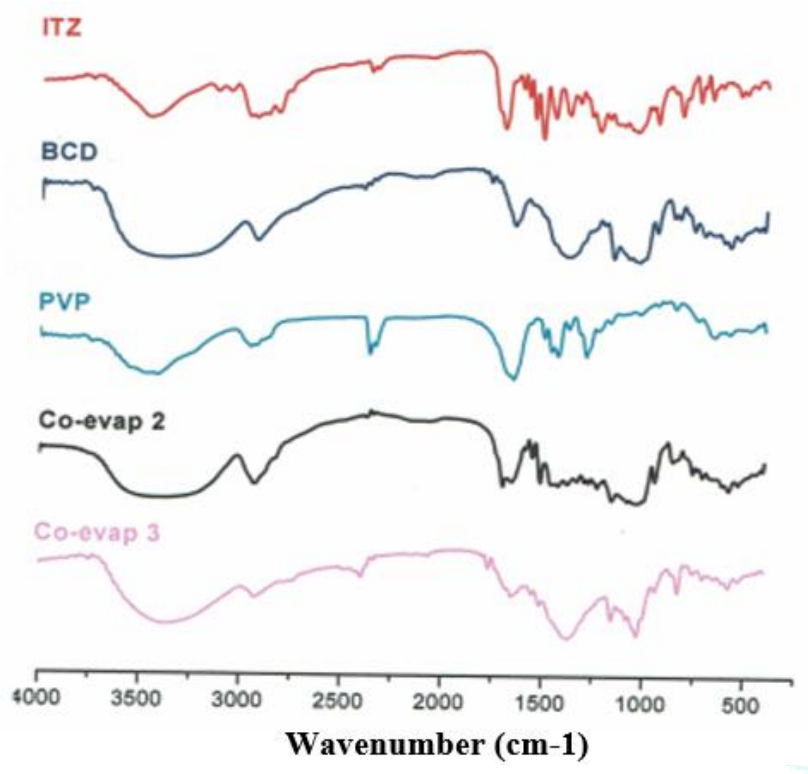

Fig.1. FT-IR spectra of pure ITZ, $\beta$-CD, PVP, $\beta$-CD-ITZ inclusion complex (Co-evap 2 ) and $\beta$-CD-PVP- ITZ inclusion complex (Co-evap 3).

\section{DSC Analysis}

We note that, within the decomposition range of CD lattice, the physical characteristics of the guest molecules such as boiling, melting and sublimation point either get shifted or disappeared (10). The DSC thermograms of pure drug, $\beta-C D$, PVP-K30, binary and ternary inclusion complexes are illustrated in Fig.2. In the DSC curve of the pure drug, a small endothermic peak related to the melting point of ITZ appears at $166.49{ }^{\circ} \mathrm{C}$. Thus. The DSC thermogram of $\beta$-CD shows a broad endotherm in the region of $120-140^{\circ} \mathrm{C}$. Therefore, this fact is caused by the liberation of crystal water molecules from the cavity (11). From the other hand, the binary and ternary inclusion complexes thermograms reveal an endothermic drug peak with less sharpness and intensity compared to the drug pure. This last, represents a strong indication of the true complex of ITZ with $\beta$-CD formation. Hence, PVP may partially convert to the amorphous state and molecularly dispersed inside the cavity of $\beta$-CD

\section{X-RD Studies}

P-XRD is a useful tool for the detection of $\beta$-CD complexation in powder or microcrystalline states. XRD studies could be employed to detect any change of crystallinity of a compound upon host-guest interactions (19). The X-ray diffraction patterns of drug, $\beta$-CD, PVP-K30, binary and ternary systems are presented in Fig. 3. P-XRD of ITZ exhibited characteristic peaks at $14.2^{\circ}, 17.2^{\circ}, 17.7^{\circ}, 22.1^{\circ}, 26.3^{\circ}, 33.4^{\circ}$ and $37.4^{\circ}(2 \theta)$ that were intense and sharp, indicating its crystalline nature (20). The XRD pattern of $\beta$-CD revealed several sharp and intense diffraction peaks, which are indicative of its crystalline character. The complete absence of any diffraction peaks for PVP-K30 demonstrated its amorphous nature. The obtained patterns reveal that the drug crystallinity of binary and ternary inclusion complexes was affected significantly indicating that Iitraconazole was present in the amorphous state in all these formulations.

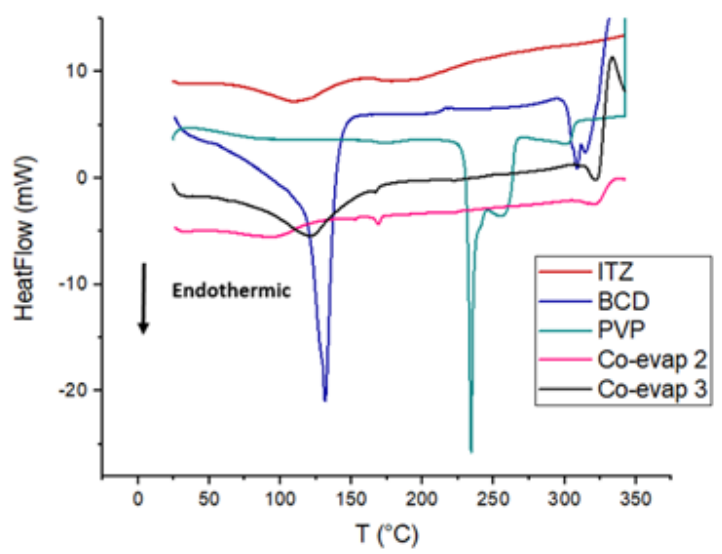

Fig.2. DSC curves of pure ITZ, $\beta$-CD, PVP, $\beta$-CD-ITZ inclusion complex (Co-evap 2) and $\beta$-CD-PVP- ITZ inclusion complex (Co-evap 3).

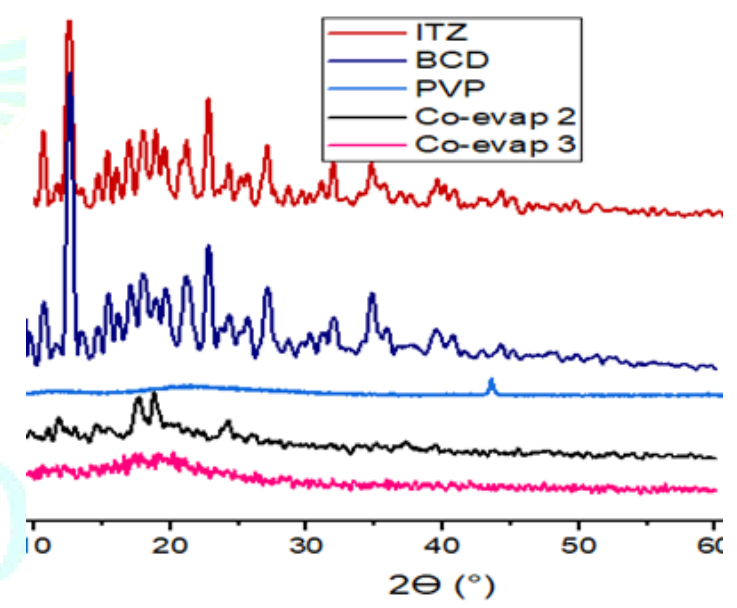

Fig.3. X-Ray diffractograms of pure ITZ, $\beta$-CD, PVP, $\beta$-CD-ITZ inclusion complex (Co-evap 2) and $\beta$-CD-PVP- ITZ inclusion complex (Co-evap 3).

\section{In Vitro Drug Release Study}

In Fig4, the dissolution profiles of pure ITZ, binary and ternary inclusion complexes in $0.1 \mathrm{~N} \mathrm{HCl}$ (with $\mathrm{pH} 1.2$ ) are depicted.

The accelerated ITZ release rate from ternary inclusion complexes could possibly due to the partial entrapment of drug molecules inside the $\beta$-CD torus. It could impart hydrophilic character and eventually increase the solubility and wettability of ITZ. The improvement in dissolution rate might also be due to the conversion to the high energetic amorphous state and reduction in crystallinity of ITZ complexation in co-evaporation method, which was verified from FTIR, DSC and XRD studies. In fact, the binary complexes exhibit lower dissolution profiles compared with the ternary systems. This is due to a greater inclusion and improvement of complexation efficiency of $\beta$-CD in the presence of PVP-K30. 


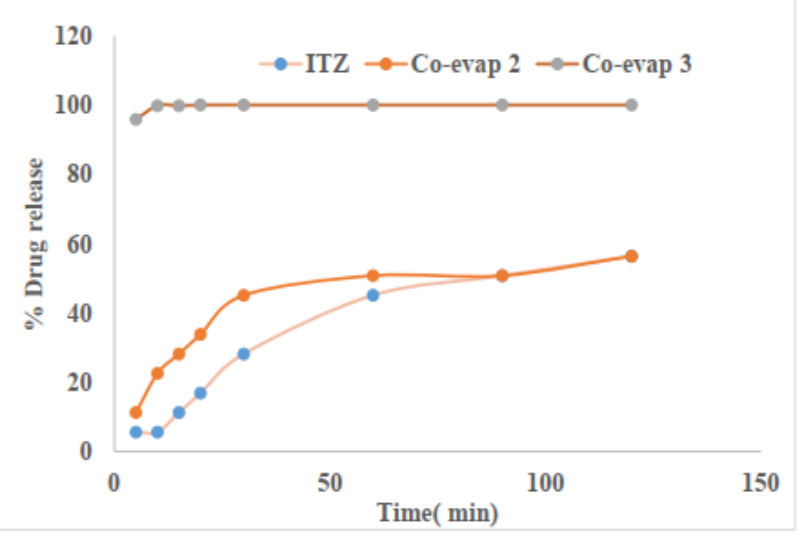

Fig.4. In vitro dissolution profile of pure ITZ, $\beta$-CD-ITZ inclusion complex (Co-evap 2) and $\beta$-CD-PVP- ITZ inclusion complex (Co-evap 3).

\section{CONCLUSION}

A significant enhancement of the solubility and bioavailability of the inclusion complexes of ITZ and $\beta$-CD in the presence and absence of hydrophilic polymer (PVP-K30) are successfully obtained. The results of the study illustrate the formation of ITZ- $\beta$-CD inclusion complexes at a 1:2 M ratio in solution. The FTIR studies deny the evidence of chemical interactions between the drug and carriers. DSC and XRD studies confirmed partial morphism of the drug after inclusion complexation. Inclusion complexes shows significantly higher drug dissolution rate compared with pure drug. The use of $\beta-C D$ in junction with PVP-K30 improves the drug solubility and dissolution rate by increasing the complexation efficiency of $\beta$-CD. The final formulation that incorporates ternary complex of ITZ demonstrates acceptable physical properties and greater release profile compared to tablets containing pure ITZ. Thus, the strategy of addition of hydrophilic polymer (PVP$\mathrm{k} 30$ ) to ITZ- $\beta$-CD complexes may provide a promising way to enhance the solubility and dissolution characteristics of drug. Hence, the obtained result may offer promoting advantages of reducing the dose of the drug and the quantity of the needed $\beta$-CD. Moreover, hydrophilic polymer associated ITZ- $\beta$-CD systems constitute a new drug delivery system, that considerably improves the therapeutic benefits of ITZ for antifungal infection treatment.

\section{REFERENCES}

1. De Beule K, Van Gestel J. Pharmacology of Itraconazole. Drugs. 1 déc 2001; 61(1):27-37.

2. Neslihan Gursoy R, Benita S. Self-emulsifying drug delivery systems (SEDDS) for improved oral delivery of lipophilic drugs. Biomedicine \& Pharmacotherapy. 1 avr 2004; 58(3):173-82.

3. Lakshman JP, Cao Y, Kowalski J, Serajuddin ATM. Application of Melt Extrusion in the Development of a Physically and Chemically Stable High-Energy Amorphous Solid Dispersion of a Poorly Water-Soluble Drug. Mol Pharmaceutics. 1 déc 2008; 5(6):994-1002.
4. Da Costa MA, Seiceira RC, Rodrigues CR, Hoffmeister CRD, Cabral LM, Rocha HVA. Efavirenz Dissolution Enhancement I: Co-Micronization. Pharmaceutics. mars 2013; 5(1):1-22.

5. Ren F, Jing Q, Tang Y, Shen Y, Chen J, Gao F, et al. Characteristics of Bicalutamide Solid Dispersions and Improvement of the Dissolution. Drug Development and Industrial Pharmacy. 1 janv 2006; 32(8):967-72.

6. Blagden N, de Matas M, Gavan PT, York P. Crystal engineering of active pharmaceutical ingredients to improve solubility and dissolution rates. Advanced Drug Delivery Reviews. 30 juill 2007; 59(7):617-30.

7. Dewaard H, Hinrichs WLJ, Visser MR, Bologna C, Frijlink HW. Unexpected differences in dissolution behavior of tablets prepared from solid dispersions with a surfactant physically mixed or incorporated. International Journal of Pharmaceutics. 12 févr 2008; 349(1):66-73.

8. Wang J, Cai Z. Investigation of inclusion complex of miconazole nitrate with $\beta$-cyclodextrin. Carbohydrate Polymers. 5 mai 2008; 72(2):255-60.

9. Eid EEM, Abdul AB, Suliman FEO, Sukari MA, Rasedee A, Fatah SS. Characterization of the inclusion complex of zerumbone with hydroxypropyl- $\beta$-cyclodextrin. Carbohydrate Polymers. 1 févr 2011; 83(4):1707-14.

10. Ribeiro LSS, Ferreira DC, Veiga FJB. Physicochemical investigation of the effects of water-soluble polymers on vinpocetine complexation with $\beta$-cyclodextrin and its sulfobutyl ether derivative in solution and solid state. European Journal of Pharmaceutical Sciences. 1 nov 2003; 20(3):253-66.

11. Shanmuga Priya A, Sivakamavalli J, Vaseeharan B, Stalin T. Improvement on dissolution rate of inclusion complex of Rifabutin drug with $\beta$-cyclodextrin. International Journal of Biological Macromolecules. 1 nov 2013; 62:472-80.

12. Cevher E, Açma A, Sinani G, Aksu B, Zloh M, Mülazımoğlu L. Bioadhesive tablets containing cyclodextrin complex of itraconazole for the treatment of vaginal candidiasis. International Journal of Biological Macromolecules. 1 août 2014; 69:124-36.

13. Brewster ME, Loftsson T. Cyclodextrins as pharmaceutical solubilizers. Advanced Drug Delivery Reviews. 30 juill 2007; 59(7):645-66.

14. Sarah G, Robert CS. 2-Hydroxypropyl- $\beta$-cyclodextrin (HP- $\beta$ CD): a toxicology review. Food and Chemical Toxicology. 2005; 43:1451-1459.

15. Szejtli J. Medicinal Applications of Cyclodextrins. Medicinal Research Reviews. 1994; 14(3):353-86.

16. Anwer MK, Jamil S, Ansari MJ, Al-Shdefat R, Ali BE, Ganaie MA, et al. Water soluble binary and ternary complexes of diosmin with $\beta$-cyclodextrin: Spectroscopic characterization, release studies and anti-oxidant activity. Journal of Molecular Liquids. 1 nov 2014; 199:35-41.

17. Van-Thanh T, Vinh PVQ, Van-Hoa H. Research and Preparation of Solid Dispersion of Itraconazole in Hydroxypropyl-BetaCyclodextrin. In: Toi VV, Lien Phuong TH, éditeurs. 5th International Conference on Biomedical Engineering in Vietnam. Cham: Springer International Publishing; 2015. p. 306-10. (IFMBE Proceedings).

18. Sambasevam KP, Mohamad S, Sarih NM, Ismail NA. Synthesis and Characterization of the Inclusion Complex of $\beta$ cyclodextrin and Azomethine. International Journal of Molecular Sciences. févr 2013; 14(2):3671-82.

19. Rajashree H, Vilasrao K. Preparation and characterization of inclusion complexes of carvedilol with methylbcyclodextrin. J Incl Phenom Macrocycl Chem. 2009; 63:219-24.

20. Boldescu V, Kacso I, Borodi G, Bratu I, Duca G. Physicochemical characterization of sanguinarine-hydroxypropyl- $\beta$ cyclodextrin binary and ternary systems. J Incl Phenom Macrocycl Chem. 1 oct 2008; 62(1):143-8. 